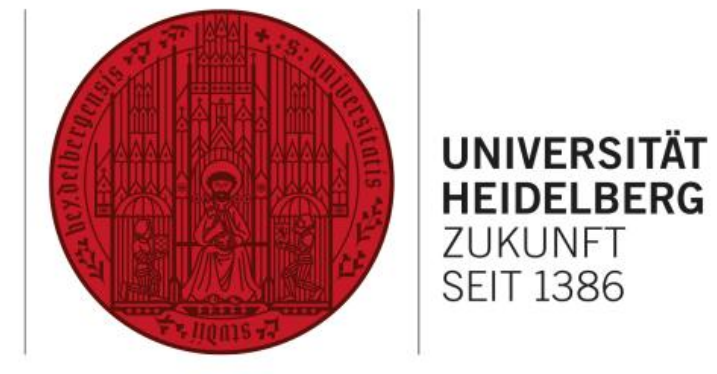

Zusammenfassung der Dissertation mit dem Titel

\title{
„Strafbarkeit grenzüberschreitend tätiger Forschungsgruppen im Bereich der biomedizinischen Forschung in der Europäischen Union“"
}

Dissertation vorgelegt von Christina Dorr

Erstgutachter: Prof. Dr. Gerhard Dannecker

Zweitgutachter: Prof. Dr. Dr. h.c. Thomas Hillenkamp

Institut für deutsches, europäisches und internationales Strafrecht und Strafprozessrecht 


\section{Strafbarkeit grenzüberschreitend}

tätiger Forschungsgruppen im

Bereich der biomedizinischen

Forschung in der Europäischen

\section{Union}

Zusammenfassung der wesentlichen Ergebnisse der Dissertation zur Erlangung der Doktorwürde der Juristischen Fakultät der Ruprecht- Karls-

Universität Heidelberg

vorgelegt von

Christina Anna Maria Dorr

Heidelberg

November 2013 
Die Arbeit zeigt die Probleme auf, die sich im Rahmen grenzüberschreitender Forschungstätigkeit aufgrund der unterschiedlichen rechtlichen Regelungen, denen die beteiligten Forscher aus verschiedenen Nationen unterliegen, ergeben. Sie konzentriert sich dabei auf die Untersuchung von Forschungsvorhaben in der Biomedizin, insbesondere der Embryonen- und Stammzellenforschung.

Die Strafbarkeitsrisiken, die sich für beteiligte Forscher aus unterschiedlichen Ländern beispielsweise bei der Teilnahme an einem europäischen Verbundprojekt ergeben können, wurden in den vergangenen Jahren insbesondere im Bereich der Stammzellen- und Embryonenforschung häufig kontrovers diskutiert.

Den Diskussionen in der juristischen Fachliteratur und in der Politik lagen dabei stets die strafanwendungsrechtlichen Regelungen der $\S \S 3 \mathrm{ff}$. StGB zugrunde, da die Anwendbarkeit nationalen Strafrechts letztlich aus ihnen herrührte. So führt beispielsweise die Regelung des $\S 9$ Abs. 2 S. 2 StGB dazu, dass deutsches Strafrecht für den im Inland tätigen Teilnehmer selbst dann gelten soll, wenn die Tat nach dem Recht der Haupttat nicht mit Strafe bedroht ist. Auch der im Ausland tätige, nach deutschem Recht verbeamtete Forscher kann sich bei Vornahme von Forschungsmaßnahmen gem. § 5 Nr. 12 StGB im Ausland nach deutschem Strafrecht strafbar machen.

Grundsätzlich ist es dem deutschen Staat nicht verwehrt, seine Strafgewalt auch über die eigenen Landesgrenzen hinaus zu erstrecken. Dies kann jedoch nicht unbeschränkt gelten. Welche Grenzen er hierbei zu beachten hat und woraus sich diese ergeben, soll die vorliegende Arbeit aufzeigen.

Der 1. Teil der Arbeit befasst sich mit den strafanwendungsrechtlichen Regelungen der $\S \S 3$ ff. StGB. Hierbei lässt sich festhalten, dass den Strafanwendungsregeln der $\S 3$ ff. StGB zugleich unrechtskonstituierende Bedeutung zukommt, wenngleich sie durch den Gesetzgeber in erster Linie unter völkerrechtlichen Aspekten eingeführt wurden. Diese völkerrechtlichen Aspekte, die eine hinreichende Verbindung des Auslandssachverhalts zum deutschen Staat sicherstellen sollen, werden im Folgenden benannt und insbesondere im 3. Teil der Arbeit näher beleuchtet.

Auf der Suche nach der Ursache der Strafbarkeitsrisiken zeigt der Blick auf die strafanwendungsrechtlichen Regelungen der $\S 3$ ff. StGB, dass die Tatbeteiligung eines 
inländischen Forschers zur Folge haben kann, dass alle Forscher, die sich an dem Projekt beteiligen, bei konsequenter Anwendung der Strafanwendungsregeln dem deutschen Strafrecht unterliegen können, ungeachtet dessen, dass sich das Vorhaben am Tatortrecht mit der dort geltenden Rechtsordnung vereinbar zeigt. Hier wird deutlich, dass es bei der zugrunde liegenden Problematik der Strafbarkeitsrisiken im Kern um die Einschränkung grundrechtlicher und europäischer Freiheitsrechte geht. Diesen Beschränkungen stehen Rechtsgüter gegenüber, deren Wertigkeit und Gefährdungsgrad in den an den Forschungsprojekten beteiligten Staaten möglicherweise unterschiedlich beurteilt werden.

Es bleibt bereits an dieser Stelle festzuhalten, dass die Untersuchung der Problematik der Strafbarkeitsrisiken unter strafanwendungsrechtlichen Aspekten zu kurz greift. Das deutsche Strafrecht darf nicht mehr als universal geltende Bewertungsnorm für jegliches Handeln über die nationalen Grenzen hinaus verstanden werden und eine weltweite Geltung beanspruchen, sondern muss sinnvoll begrenzt werden.

Hier wird die Frage nach der Strafberechtigung des deutschen Staates für solche grenzüberschreitenden Sachverhalte aufgeworfen: Was gibt dem deutschen Staat das Recht, die Geltung seines nationalen Strafrechts für eine Tat anzuordnen, die schwerpunktmäßig oder gar vollständig im Ausland stattfindet? Die aufgeworfenen Problemstellungen sollen im 5. Teil anhand der unterschiedlichen Beteiligungskonstellationen untersucht werden: Täterschaftliche Forschungsaktivität im Ausland, grenzüberschreitende Forschungstätigkeit vom Inland ins Ausland und solche vom Ausland in das Inland.

Der 2. Teil beschäftigt sich mit der Frage, wie sich diese Strafberechtigung, das ius puniendi, einordnen lässt, wo sie ihren Ursprung findet und wodurch sie begrenzt wird. Eine umfassende Strafbefugnis lässt sich nach hier vertretener Ansicht weder aus der Aufgabe noch aus dem Zweck des Strafrechts herleiten. Auch die Kategorien der Strafwürdigkeit und Strafbedürftigkeit, die sich als Ausprägungen des Verhältnismäßigkeitsgrundsatzes verstehen, zeigen sich nicht als geeignet, die Frage nach dem Bestehen der Strafbefugnis zu beantworten. Stellt man auf diese Kriterien ab und beurteilt die Frage nach der Strafberechtigung danach, ob strafwürdiges und -bedürftiges Handeln im Raum steht, so unterliegt man einem Zirkelschluss, da sich die Beurteilung nach den das materielle Unrecht bestimmenden Faktoren erst vornehmen lässt, wenn eine bestimmte Werteordnung herangezogen werden kann, die diese Frage beurteilen kann. Legt man diese Werteordnung zugrunde, hat man sich 
die Strafberechtigung jedoch bereits zuerkannt und bewertet den Sachverhalt nach eigenen nationalen Grundsätzen. Die rechtspolitisch gebotene Zurückhaltung wäre in diesem Fall verfehlt.

Im Folgenden wird deutlich, dass sich die Strafbefugnis nicht - wie zu vermuten wäre - aus der Verfassung herleiten lässt. Weder Art. 74 Nr. 1 GG noch das Rechtsstaatsprinzip konstituieren die Strafberechtigung als solche. Letztlich lässt sich festhalten, dass die Strafgewalt als Teil der Staatsgewalt verstanden werden muss und jedem Staat zukommen muss, um seine ordnungsrechtlichen Aufgaben wahrnehmen zu können und eine funktionierende Rechtsordnung schaffen und erhalten zu können.

Daran anschließend werden die Grenzen dieses Rechts aufgezeigt: Diese leiten sich in erster Linie aus der Verfassung her. In diesem Zusammenhang wird die Rechtsprechung des Bundesverfassungsgerichts zum Europäischen Haftbefehlsgesetz fruchtbar gemacht, in der das Gericht aus dem Rechtsstaatsgebot des Art. 103 Abs. 2 GG den Schutz des Vertrauens in die Verlässlichkeit der eigenen Rechtsordnung herleitet, an der der Bürger im Ausland sein Handeln ausrichten können soll. Daraus wird im Rahmen der folgenden Untersuchung ein Vertrauenssatz dahingehend entwickelt, dass die Ausdehnung der Strafgewalt ihre Grenzen in Art. 103 Abs. 2 GG finden muss, wenn sich bei einem Verhalten im Ausland ein berechtigtes Vertrauen in die Straflosigkeit nach dem Recht des Tatortstaates ausmachen lässt.

Die Grenzziehung, wann eine wesentliche Inlandsverbindung und damit ein möglicherweise berechtigtes Vertrauen besteht, erfolgt unter Zuhilfenahme der nationalen Grundrechte, insbesondere der Forschungs- und Berufsfreiheit der Art. 5 Abs. 3 und 12 GG, der europäischen Grundfreiheiten wie der Niederlassungs- und Dienstleistungsfreiheit sowie unter Rekurs auf die Grundrechte der EGRC. Hier wird untersucht wie weit die Schutzpflicht des Staates für im Ausland befindliche Rechtsgüter gehen kann. Ein verfassungsrechtliches Gebot, im Ausland begangene Grundrechtsbeeinträchtigungen nach nationalem Strafrecht zu sanktionieren wird im Folgenden für die Menschenwürde und das Recht auf Leben verneint. Im Ergebnis wird deutlich, dass sich die Frage nach dem Bestehen des ius puniendi schwerlich nur noch als allein nationale Frage ausmachen lässt. Sie hat sich vielmehr an europäischen Vorgaben messen und mit diesen in Verbindung bringen zu lassen.

Im 3. Teil werden die strafanwendungsrechtlichen Regelungen, die in den untersuchten Konstellationen Bedeutung gewinnen, einer näheren Analyse unterzogen. Die Grenzen des 
Völkerrechts, die einer unkontrollierten Erstreckung des nationalen Strafrechts auf Auslandsfälle entgegen stehen sollen, werden aufgezeigt und in diesem Zusammenhang dem völkerrechtlichen Nichteinmischungsgrundsatz besondere Beachtung geschenkt. Nach einer Übersicht über die völkerrechtlich legitimierten Anknüpfungspunkte kann als Zwischenergebnis festgehalten werden, dass eine völkerrechtskonforme Anwendung des nationalen Strafrechts nur gegeben sein kann, wenn eine hinreichend enge Verbindung des Auslandssachverhalts zu dem jeweiligen Staat in Form eines solchen Anknüpfungspunktes besteht. Es wird im Folgenden untersucht, ob eine Erstreckung der Strafgewalt im Rahmen der grenzüberschreitenden Forschung durch das Weltrechtsprinzip legitimiert sein kann mit dem Ergebnis, dass international weitgehend Einigkeit über das Verbot des reproduktiven Klonens vorherrscht. Die Frage nach der Schutzwürdigkeit des Embryos allgemein ist jedoch hoch umstritten.

Als für die zukünftige Forschung richtungsweisend wird die Entscheidung des EuGH vom 18.10.2011 über die Patentierung des menschlichen Genoms genannt, in der es zu einer verbindlichen Definition des menschlichen Embryos durch das höchste Gericht in der Europäischen Union gekommen ist.

Der 4. Teil ist der Untersuchung der tatbestandsmäßigen Beschränkung des Anwendungsbereichs des Strafrechts gewidmet und untersucht den Schutzbereich der Strafnormen. Nur unter der Voraussetzung, dass das Verhalten im Ausland inländische Interessen derart stört, dass das Strafrecht als „Ordnungsrecht“ auf den Plan gerufen werden muss, ist der Schutzbereich der nationalen Straftatbestände eröffnet. Im Kern geht es hier um die Frage des zugrunde liegenden Rechtsguts und wann das betroffene Rechtsgut dem inländischen Rechtsgüterschutz unterstellt werden soll. Nach nationaler Ansicht steht hinter der Strafandrohung nach dem ESchG im Wesentlichen der Schutz der Menschenwürde und des menschlichen Lebens; als problematisch stellt sich jedoch erneut die fehlende einheitliche Aussage zur Schutzwürdigkeit des menschlichen Embryos dar.

Zur Frage der Prüfungsreihenfolge der Punkte Strafanwendungsrecht und Bestimmung des Schutzbereichs wird einer in der Literatur teilweise vertretenen Ansicht gefolgt, die dem Strafanwendungsrecht Vorrang vor der Frage nach der Schutzbereichsprüfung einräumt.

Der 5. Teil setzt sich mit der Frage auseinander, wie die unterschiedlichen denkbaren Konstellationen im Rahmen der grenzüberschreitenden Forschungstätigkeit, insbesondere der 
täterschaftlichen Forschung und Beteiligung, und die damit einhergehenden rechtlichen Probleme eingeordnet werden können.

Hier wird zunächst die Forschungsaktivität, die komplett im Ausland vorgenommen wird, untersucht. Bevor es zur Anwendung deutschen Strafrechts auf einen Auslandssachverhalt kommen kann, stellt sich die Frage nach dem Bestehen des ius puniendi. Ob dieses für den jeweiligen Sachverhalt mit Auslandsberührung bestehen kann oder nicht soll unter Rekurs auf die zentralen Aussagen zur Untersuchung der Entscheidung des BVerfG zum Europäischen Haftbefehlsgesetz bewertet werden. Aus dem Rechtsstaatsprinzip des Art. 103 Abs. 2 GG lassen sich Grenzen herleiten, die bei der Frage nach dem Bestehen der Strafberechtigung Beachtung finden müssen. Die Rechtsprechung des BVerfG zum hinreichenden Inlandsbezug wird fortgeführt: So soll neben dem Vertrauen bei reinen Inlandssachverhalten allein der inländischen Strafgewalt zu unterliegen, auch das Vertrauen, bei reinen Auslandssachverhalten der inländischen Strafgewalt gerade nicht mehr zu unterliegen, geschützt sein und sich aus Art. 103 Abs. 2 GG ableiten lassen.

Im Rahmen dieser Untersuchung zeigt sich auch, dass ein Forschungsvorhaben in der Europäischen Union grundsätzlich unter den Schutz der europäischen Grundfreiheiten fällt und der Erstreckung der nationalen Strafgewalt bereits aufgrund des Anwendungsvorrangs des Unionsrechts im Konfliktfall Grenzen gesetzt sein können.

Die Androhung der Strafbarkeit der entsprechenden Verhaltensweisen setzt sich neben den Grundfreiheiten auch zu den national wie europäisch garantierten Grundrechten der Forschungs- und Berufsfreiheit in Widerspruch. Für den Forscher im Ausland muss gelten, dass er aufgrund der vollständigen Vornahme aller Forschungshandlungen im Ausland im Einklang mit dortigem Recht einen maßgeblichen Auslandsbezug geschaffen hat, der es legitimiert, dass er grundsätzlich darauf vertrauen können darf, sein Handeln an dem Recht am Handlungsort auszurichten und nicht mehr dem deutschen Recht zu unterliegen.

Im Rahmen der Ausführungen zum Strafanwendungsrecht werden im Folgenden die Regelungen des $§ 5$ Nr. 12 und 13 StGB näher beleuchtet. Als Ergebnis bleibt hier festzuhalten, dass das aktive Personalitätsprinzip, wie es $\S 5$ Nr. 12 1. Var. StGB zugrunde liegt, sich aufgrund des Verzichts auf die identische Tatortnorm als unvereinbar mit dem Völkerrecht zeigt und daher nicht herangezogen werden kann, um die Berechtigung zur Bestrafung zu begründen. Hierin liegt eine völkerrechtswidrige Intervention. 
Dagegen wird dem Staatsschutzprinzip eine hinreichende legitimierende Wirkung zugesprochen. Ein Anknüpfen an dieses Prinzip und eine damit einhergehende Erstreckung der Strafgewalt kann im Rahmen von $§ 5$ Nrn. 12 und 13 StGB jedoch nur in Betracht kommen, wenn ein hinreichender Bezug zur dienstlichen Tätigkeit auch tatsächlich besteht und damit ein staatliches Schutzinteresse in Gefahr zu sein scheint.

Weitere Voraussetzung für die Strafbarkeit nach deutschem Strafrecht ist, dass das betroffene Rechtsgut auch dem inländischen Rechtsgüterschutz unterfällt.

Daran anschließend wird die Konstellation der ,grenzüberschreitenden Forschung vom Inland aus in das Ausland“ untersucht. Hierunter fallen Forschungsmaßnahmen, die schwerpunktmäßig im Ausland stattfinden, jedoch vom Inland aus in irgendeiner Weise unterstützt werden. Zunächst wird die inländischer Teilnahme an ausländischer und nach deutschem Recht verbotener Forschung, die sich nach § 9 Abs. 2 S. 2 StGB bestimmt, näher beleuchtet. Nach einer umfassenden Untersuchung der Regelung des $§ 9$ Abs. 2 S. 2 StGB und ihrer Vereinbarkeit mit dem Akzessorietätsgrundsatz wird aufgezeigt, dass sich die strafanwendungsrechtlichen Regelungen nicht geeignet zeigen, die Problematik der Strafbarkeitsrisiken bei grenzüberschreitender Forschung zu lösen.

Gleiches gilt für die sich daran anschließende Untersuchung der täterschaftlichen Beteiligung an grenzüberschreitenden Forschungsprojekten im Ausland nach $\S 9$ Abs. 1 StGB. Es wird dargelegt, dass es hier nicht - wie in der Literatur teilweise vertreten - um die Zurechnung von Handlungsorten gehen kann.

Vielmehr zeigt sich, dass die Akzessorietäts- und die Zurechnungsproblematik nur von untergeordneter Bedeutung sind und letztlich die Frage nach dem ius puniendi und seinen Grenzen im Mittelpunkt steht, die unabhängig von einer dogmatischen Einordnung in Teilnahme oder Mittäterschaft beurteilt werden muss.

Deshalb wird auch im Rahmen dieser Konstellation auf Art. 103 Abs. 2 GG sowie auf die europäischen Grundfreiheiten und die Forschungs- und Berufsfreiheit zurückgegriffen. Als Ergebnis lässt sich festhalten, dass der deutsche Forscher, der sich im Ausland an einem Forschungsprojekt beteiligt, grundsätzlich darauf vertrauen können muss, straflos zu sein. Hingegen steht für den Forscher, der sich vom Inland aus an einem Forschungsprojekt im Ausland beteiligt, sein tatsächlich erbrachter Tatbeitrag im Mittelpunkt: Je größer die Bedeutung ist, die die Handlung des Forschers im Inland für das Gelingen des Forschungsvorhabens hat und je mehr diese zur Rechtsgutsverletzung beiträgt, desto eher besteht im Inland die Berechtigung dieses Verhalten zu bestrafen. 
Unter völkerrechtlichen Aspekten stellen sich die Regelungen des $§ 9$ Abs. 1 bzw. Abs. 2 S. 2 StGB als weniger problematisch dar, als dies im Rahmen des $§ 5$ Nrn. 12 und 13 StGB der Fall war. Zwar wird auch hier auf eine identische Tatortnorm verzichtet. Anders als im Rahmen der ersten Konstellation kommt es jedoch im Rahmen des § 9 StGB nicht zu einer Anwendung deutschen Strafrechts im Ausland. Da sich der Beteiligte im Inland befindet, lässt sich die strafrechtliche Erfassung seiner Tat auf das Territorialitätsprinzip stützen. Anders für den Forscher im Ausland, zu dessen Strafbarkeit die überwiegende Ansicht der Lehre durch Zurechnung kommen mag: Die Begründung eines Handlungsortes im Inland durch Zurechnung und die damit einhergehende Erstreckung der Strafgewalt in das Ausland kommt einer Missachtung fremder Souveränität gleich, da sie ausländische Anschauungen und Wertungen, das entsprechende Verhalten nicht unter Strafe zu stellen, nicht achtet.

Die dritte Konstellation widmet sich der grenzüberschreitenden Forschungsaktivität vom Ausland in das Inland. Auch hier soll der Problematik nicht anhand der Kategorisierung in Mittäterschaft oder Teilnahme begegnet werden, sondern die Frage nach dem Bestehen der Strafberechtigung im Mittelpunkt stehen. Abzustellen ist hier insbesondere auf den Schwerpunkt der Tat und die Natur des Tatbeitrags. Ist die Handlung nur von untergeordneter Bedeutung für den Erfolg des Projekts, so liegt nahe, dass die Strafberechtigung für den deutschen Staat in diesem Fall fehlt.

Der 6. Teil widmet sich der Erarbeitung eines Lösungsvorschlags. Nachdem ein Rückgriff auf die früher geltende Norm des $\S 3$ Abs. 2 a.F. StGB nicht in Betracht kommt, da der Gesetzgeber mit ihrer Streichung bewusst auf diese Regelung verzichtet hat, und auch die prozessuale Lösung über $§ 153$ c StPO als nicht ausreichend erachtet wird, soll eine Lösung in der Beachtung des ausländischen Rechts versucht werden.

Das Berufsrecht anderer Staaten soll eine Grenze der Strafbarkeit bilden, die in die Frage nach der Strafberechtigung einfließen muss und die im Rahmen der europäischen Grundfreiheiten und Grundrechte Berücksichtigung finden muss.

Klargestellt wird in diesem Zusammenhang auch, dass dies keineswegs dazu führen darf, den nationalen Schutzstandard aufzuweichen und nationale Werte preis zu geben. An der innerstaatlichen Werte- und Normenordnung soll sich nichts ändern, entscheidend ist allein, durch hinreichende Beachtung der Frage nach dem Bestehen des ius puniendi das 
Strafbarkeitsrisiko bei der Beteiligung an grenzüberschreitenden Forschungsprojekten zu minimieren. Durch den hier vorgeschlagenen Lösungsweg soll es weder zu einer Anwendung ausländischen Rechts noch zu einer Billigung von Rechtsgutsverletzungen im Ausland unter dem Deckmantel der Grundfreiheiten bzw. der Forschungsfreiheit kommen.

Auch soll diese Vorgehensweise keinesfalls dazu führen, dass für einige Mitgliedstaaten gänzlich unerträgliche Vorgehensweisen gemeinschaftlich gefördert werden.

Ziel ist lediglich, die Frage nach der Strafrechtsanwendung zu überdenken und nicht durch die strikte Anwendung der $\S \S 3$ ff. StGB das deutsche Strafrecht unreflektiert zur Anwendung zu bringen. Der Frage nach dem Bestehen des ius puniendi in Verbindung mit der Untersuchung des betroffenen Rechtsguts und ihre Verträglichkeit mit Europa- wie Verfassungs- und nicht zuletzt mit dem Völkerrecht, soll verstärkt Gewicht verschafft werden.

Zentrale Bedeutung erlangen daher zu setzende Grenzen der Straflosigkeit. Hierzu wird es erforderlich sein, allgemein anerkannte und übergreifend geregelte Standards herauszuarbeiten, Verhaltensweisen also, die weder durch die Berufs- oder Forschungs-, noch durch die Grundfreiheiten gerechtfertigt sein können. Letztlich kommt daher auch der hier erarbeitete Lösungsvorschlag nicht ohne übergreifende Regelungen und damit nicht ohne die Zuhilfenahme europäischer oder internationaler Organisationen aus.

Im 7. Teil schließt die Arbeit mit einer Zusammenfassung und einem Ausblick. Hier bleibt festzuhalten, dass, um letztlich für mehr Rechtssicherheit auf dem Gebiet der biomedizinischen Forschung sorgen zu können, als einzig praktikable Lösung für die Zukunft die Schaffung international abgestimmter Regelungen auf diesem Gebiet bleibt. Da der Europäischen Union hierzu mangels Kompetenzen nur bedingt Möglichkeiten zur Verfügung stehen, wäre ein (Wieder-)Tätigwerden des Europarates wünschenswert. Die Arbeit ist mit dem Zusatzprotokoll zum Übereinkommen über Menschenrechte und Biomedizin über biomedizinische Forschung aus dem Jahre 2005 zwar bereits auf den Weg gebracht. Gerade, weil dieses jedoch den heiklen Bereich der Embryonenforschung ausklammert, besteht auf diesem Gebiet noch immer dringender Handlungsbedarf, um die grenzüberschreitende Forschung zu erleichtern.

Bis dies gelingen wird, wird die Teilnahme an grenzüberschreitenden Forschungsprojekten für die Wissenschaftler weiterhin mit einem Strafbarkeitsrisiko behaftet bleiben. Die 
vorliegende Untersuchung zeigt, dass man der Problematik mit der Beachtung der Frage nach dem Bestehen der Strafgewalt begegnen und eine reflektierte Anwendung des Strafanwendungsrechts unter ergänzender Beachtung materieller Aspekte zu durchaus sinnvollen und angemessenen Ergebnissen führen kann, die dem ausländischen Recht hinreichend Rechnung tragen. 